\title{
Cultura ambiental para mitigar la contaminación de las microcuencas proveedoras de agua a la ciudad de Loja
}

\section{Environmental culture to mitigate the contamination of water supply micro- basins in the city of Loja}

Aurita Geovania Gonzaga Figueroa

Universidad Nacional de Loja, Ecuador

Autor para correspondencia: aurita.gonzaga@unl.edu.ec

Fecha de recepción: 23 de Agosto de 2017 - Fecha de aceptación: 30 de Septiembre de 2017

Resumen: La formación de la cultura ambiental en la población constituye una alternativa para mitigar impactos ambientales. Se interviene científicamente con un proyecto de intervención comunitaria que permitió desarrollar estrategias de mitigación de impactos contaminantes ambientales. El objetivo de la investigación fue diseñar un proyecto de intervención para contribuir a la formación de la cultura ambiental de los pobladores de la Microcuenca de Jipiro de la hoya de Loja, partiendo de la demostración de la existencia del problema, y la toma de conciencia por parte de los líderes formales y habitantes de la región, el mismo se aplica a partir de la metodología del autodesarrollo comunitario que propone un proceso de participación activa de los implicados en la determinación y solución del problema, en este caso la población local y sus líderes formales e informales. La evaluación de los resultados del proyecto de intervención para contribuir a la formación de la cultura ambiental de los pobladores corrobora la hipótesis general planteada, indican la toma de conciencia de los habitantes sobre los problemas de su entorno, específicamente la problemática del agua y la contaminación de la microcuenca investigada, en la cual se está produciendo un proceso de contaminación que amenaza la salud y la propia convivencia hombre con la naturaleza y entre los propios hombres. Durante el proceso de intervención se desarrollaron talleres que permitieron concienciar a los participantes del problema ambiental de su comunidad, dado que la formación de la cultura ambiental se convierte en un proyecto de carácter ecológico y socio-político, no se limita a atenuar los problemas ambientales producidos por el desarrollo depredador de recursos naturales, está orientado a la promoción de sus transformaciones cualitativas en la conciencia social. Desde la perspectiva del autodesarrollo comunitario se evidencian cambios favorables en cuanto la conciencia crítica, la identificación de las contradicciones principales que se expresan entre la situación real de deterioro de la microcuenca y la cultura ambiental de los pobladores para solucionar este deterioro. Se aprecia la disposición a la participación en las tareas para solucionar los problemas actuales e impedir nuevos problemas, esta participación se sustenta en procesos de reflexión.

Palabras claves: autodesarrollo comunitario; cultura ambiental; cuencas hidrográficas; impacto ambiental

Abstract: To instill an environmental culture in the population constitutes an alternative to mitigate environmental impacts. It participates scientifically with a community intervention project that allowed the development of strategies to mitigate environmental pollution impacts. The objective of the research was to design an intervention project to contribute to the formation of the environmental culture of the inhabitants of the Jipiro Micro-watershed of the Loja basin, starting from the demonstration of the existence of the problem, and the awareness of the formal leaders and inhabitants 
of the region, this is applied from the methodology of community self-development that proposes a process of active participation of those involved in the determination and solution of the problem, in this case the local population and their formal and informal leaders. The evaluation of the results of the intervention project to contribute to the formation of environmental culture of the inhabitants corroborates the stated general hypothesis, indicate the awareness of the inhabitants about the problems of their environment, specifically the water problem and pollution of the investigated micro-watershed, in which a process of pollution is occurring that threatens the health and the very coexistence of man with nature and among the men themselves. During the intervention process, workshops were held to raise awareness among participants of the environmental problem of their community, since the formation of an environmental culture becomes an ecological and socio-political project, is not limited to mitigate environmental problems produced by the predatory development of natural resources, is oriented to the promotion of its qualitative transformations in the social conscience. From the perspective of community self-development, there are favorable changes in terms of critical awareness, identification of the main contradictions expressed between the actual deterioration situation of the micro-watershed and the environmental culture of the inhabitants to solve this deterioration. The willingness to participate in the tasks to solve the current problems and to prevent new problems is appreciated, this participation is based on processes of consideration.

Key words: community self-development; environmental culture; watersheds; environmental impact

\section{Introducción}

La formación de la cultura ambiental en la población constituye una alternativa para mitigar impactos ambientales. Se reconoce que la cultura ambiental resultado de la educación ambiental que el hombre ha ido recibiendo durante toda su existencia, evidenciada en la transformación de su conciencia social e individual; del sistema de conocimientos, sentimientos, actitudes y valores relacionados con la problemática ambiental; y su expresión en las relaciones entre los seres humanos, de estos con la sociedad, y de esta con la naturaleza. La formación de la cultura ambiental desde lo comunitario es un proceso que en su génesis puede contribuir a los procesos emancipatorios de la sociedad (Sato: 2002, Roque 2003, UNCSD 2016).

Los principales problemas vinculados al aprovechamiento y manejo de los recursos hídricos en el sur del Ecuador, tiene relación con las características particulares de los factores biofísicos y socioeconómicos. El relieve abrupto, suelos frágiles fácilmente erosionables, estacionalidad marcada de la precipitación con lluvias de elevada intensidad y fuertes sequías de varios meses, avance de la frontera agrícola en áreas no aptas, deforestación acelerada y mal manejo del bosque y vegetación protectoras, mal uso del agua de riego, uso inapropiado de agroquímicos, predominio del minifundio, niveles bajos de organización comunitaria, ausencia de políticas y estrategias que privilegien el manejo de las cuencas altas abastecedoras de agua potable para las poblaciones de la Ciudad de Loja.(Boff, 2004; Gonzaga,2014).

Los elementos antes mencionados han contribuido a la erosión, al deterioro permanente de los ecosistemas naturales, superficiales y de subsuelo, de la fertilidad del suelo y por ende a la inestabilidad de las tierras, que tiende a agudizarse debido al proceso de construcción de ciudadelas en las últimas décadas. Del producto que queda, de los materiales de construcción tales como arcilla, arena, grabas trituradas, se genera otra problemática ambiental visible, cual es la afectación del suelo por disposición inadecuada de residuos sólidos insolubles. (Programa de 
las Naciones Unidas para el Medio Ambiente, Municipalidad de Loja y Naturaleza y Cultura Internacional. 2007)

Una de las alternativas más importantes para mitigar los impactos de los problemas ambientales está en el proceso de educación de la ciudadanía, es por ello que en la presente investigación se plantea como problema la siguiente cuestión: ¿Cómo contribuir a la formación de la cultura ambiental de los pobladores de las comunidades para mitigar los impactos ambientales en la Microcuenca del Rio Jipiro de la hoya de Loja?

La experiencia de intervención realizada tuvo por objetivo general formar una cultura ambiental en los pobladores de las comunidades para potenciar el desarrollo de la conciencia crítica de la ciudadanía para mitigar la contaminación ambiental en las microcuencas estudiadas. Sustentado en este propósito, se formuló la siguiente hipótesis: el impacto ambiental causado por la contaminación a las microcuencas de la hoya y ciudad de Loja se debe a la acción irresponsable de diferentes actividades humanas, y se podría mitigar a través de la formación de la cultura ambiental en los pobladores de las comunidades.

Como se puede apreciar el enfoque que asumo está dirigido a concebir la formación de la cultura ambiental de la comunidad insertada en el entorno social como alternativa para mitigar los impactos ambientales que se están produciendo en el entorno. Para ellos es necesario profundizar en la concepción teórica sobre el trabajo comunitario que se asume.

La formación de la cultura ambiental desde lo comunitario es un proceso que en su génesis puede contribuir a los procesos emancipatorios de la sociedad. Se reconoce que la cultura ambiental resultado de la educación ambiental que el hombre ha ido recibiendo durante toda su existencia, evidenciada en la transformación de su conciencia social e individual; del sistema de conocimientos, sentimientos, actitudes y valores relacionados con la problemática ambiental; y su expresión en las relaciones entre los seres humanos, de estos con la sociedad, y de esta con la naturaleza. (Teixeira y Urías, 2008).

\section{Metodología}

\section{Área de Estudio}

La investigación se desarrolló en la Microcuenca de Jipíro ubicada en la parroquia de San Juan del Valle del cantón y provincia de Loja, que forman parte de la zona de amortiguamiento del Parque Nacional Podocarpus. Tiene una superficie aproximada de 3200 ha, se descompone en tres minicuencas: Sangre, Volcán y El Salado. Con una altura 2059-3086 msnm. Precipitación media anual de $851 \mathrm{~mm}$ y temperatura media anual de $15,5^{\circ} \mathrm{C}$. Produce $200 \mathrm{l} / \mathrm{s}$ y provee el $20 \%$ del agua de la demanda total de la población de la ciudad de Loja. El clima en el lugar es típico de una zona de páramo, (temperado-ecuatorial-subhúmedo con altas cantidades de precipitaciones en el año como lo es en toda la hoya de Loja. 
MAPA DE USO ACTUAL DEL SUELO DE LA MICROCUENCA JIPIRO
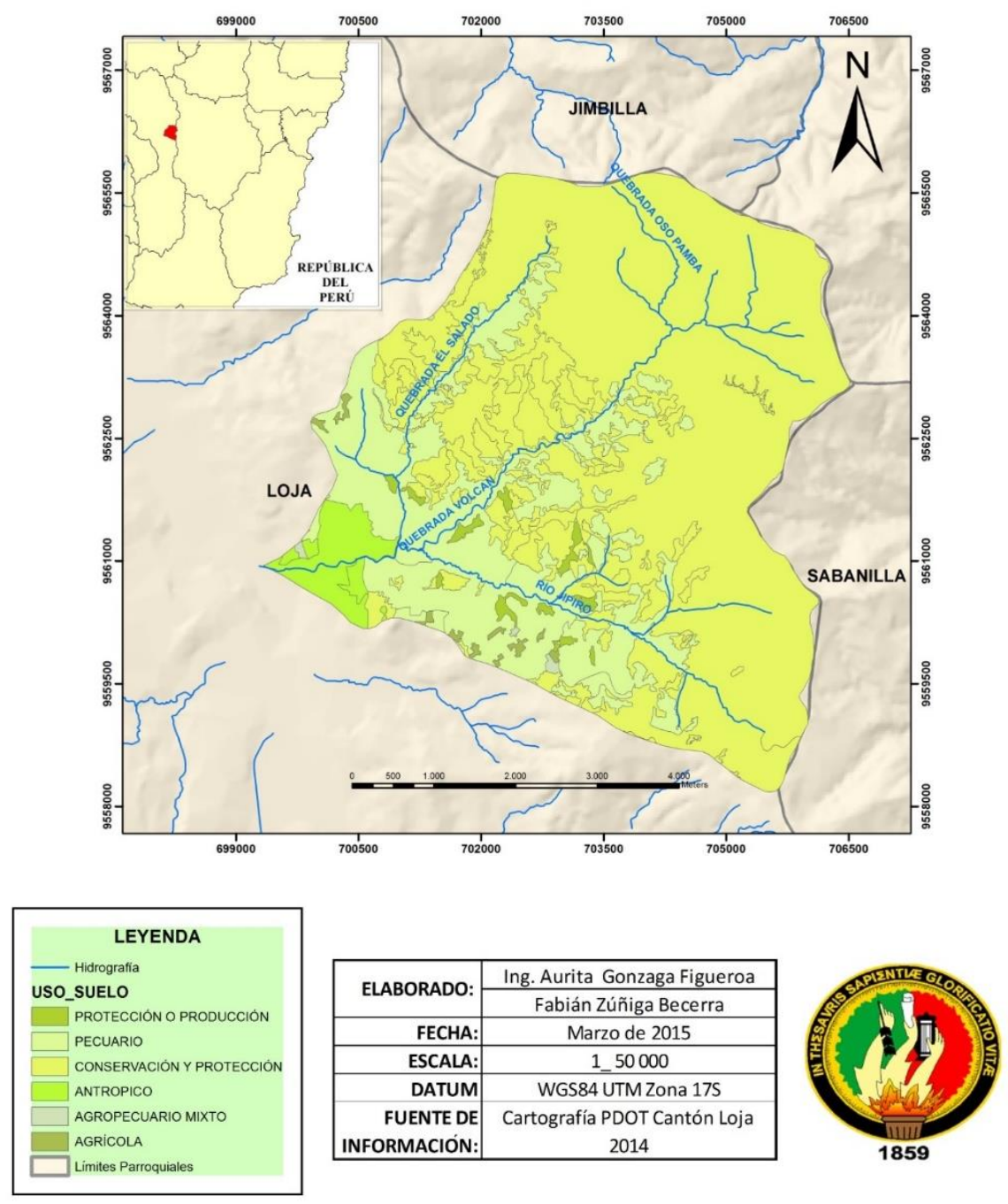

Metodología de la investigación:

La investigación desde el punto de vista metodológico es cuantitativa y cualitativa dado que emplea métodos que tributan a ambas perspectivas metodológicas a través de procesos inductivos y deductivos, con el uso de las herramientas participativas de investigacion-accionparticipacion (IAP). El trabajo se sustentó en la metodología del autodesarrollo comunitario (MAC), que comprende 5 etapas (Primera etapa: intercambio inicial con el sujeto demandante o necesitado de la acción profesional, Segunda etapa: exploración del escenario y formulación del pre diagnóstico, Tercera etapa: proceso en sí de diagnóstico y búsqueda de soluciones, Cuarta etapa: evaluación, Quinta etapa: sistematización) que son las que organizan la lógica del proceso investigativo (Colectivo de Autores, 2004).

Cada una de las etapas antes descritas están sustentadas por diferentes métodos de investigación científicos, los cuales orientan el proceso de investigación y permiten analizar, 
sintetizar y descubrir la realidad objeto de estudio, llevando a cabo un estudio minucioso y claro de la problemática existente, las cuales se presentan y describen a continuación.

Primera etapa: intercambio inicial con el sujeto demandante o necesitado de la acción profesional.

Para ello, se realizaron entrevistas en profundidad a los líderes formales de las comunidades del barrio Jipiro Alto.

\section{Segunda etapa: exploración del escenario y formulación del pre-diagnóstico.}

Se aplicaron los métodos de observación participante y aplicación de encuestas (Anexo 1) a la comunidad, mediante la cual se pudo diagnosticar los malestares y contradicciones que presentaron los habitantes de la microcuenca objeto de intervención.

\section{Tercera etapa: proceso en sí de diagnóstico y búsqueda de soluciones.}

Se realizó a través de los espacios de reflexión grupal y del uso de dispositivos grupales, como los grupos de reflexión, discusión y focal, los cuales proporcionaron la posibilidad de que la comunidad participe e identifique las contradicciones que constituyen causas de sus malestares, y formulen alternativas que promuevan el desarrollo.

\section{Cuarta etapa: capacitación y evaluación.}

Se realizaron un total de cinco talleres (Anexo 2) donde se desarrollaron los principales problemas identificados en las etapas anteriores, con el propósito de buscar el impacto transformador en la realidad comunitaria. En cada taller se evaluó los niveles de autodesarrollo logrados para problemáticas comunitarias, así como los cambios de conductas logrados a nivel individual y colectivo y la apropiación crítica y autocrítica, el compromiso alcanzado y la decisión para proteger y cuidar la vegetación, agua y suelo, manejar adecuadamente los desechos sólidos y mantener los ríos limpios.

\section{Quinta etapa: sistematización.}

Se sistematizó las experiencias básicamente a través de la revisión de las evaluaciones de los procesos grupales y de la reflexión teórica de los resultados obtenidos.

\section{Resultados}

Para el desarrollo de la problemática ambiental del área de estudio, se realizaron encuestas a 180 habitantes del barrio de Jipiro Alto. Teniendo en cuenta las respuestas obtenidas, se observa que los pobladores evaluados tienen conciencia de la problemática ambiental en relación a la contaminación de las microcuencas, así como la responsabilidad que ellos mismos tienen sobre dicha problemática (Figura 1). En lo que respecta a las posibles soluciones, los habitantes conocen qué medidas de control están en sus manos para enfrentar la problemática (Figura 2), aunque el total de ellos considera que no están preparados para ello. Considerando las 
relaciones entre la contaminación de los ríos y la salud humana, el 54\% de los encuestados aseguran no tener ningún conocimiento de los daños que dicha contaminación puede tener sobre la salud humana, aunque el $66 \%$ considera que los vecinos que viven muy próximos a los ríos sí tienen algún conocimiento, aunque escaso. Ante la pregunta de si están dispuestos a cambiar dicha situación, el 90\% de los encuestados respondieron afirmativamente, y el total opina que las medidas que el Municipio debería tomar para mitigarla son mingas y campañas.

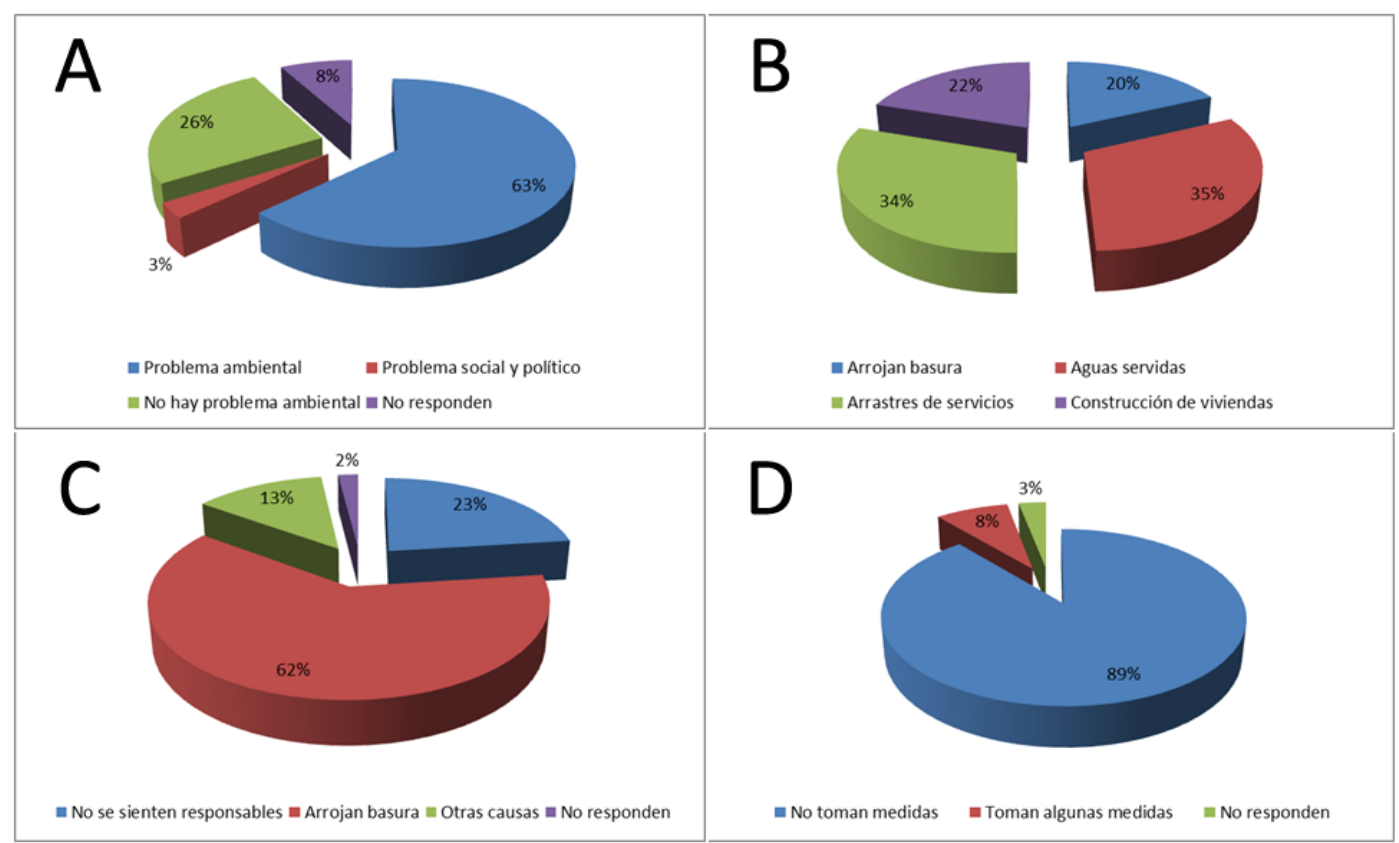

Figura 1. Proporciones de respuestas obtenidas a las siguientes preguntas: A) ¿Qué opina usted sobre la contaminación de las microcuencas? B) ¿Cómo ocurre el proceso de contaminación de las microcuencas por parte de los pobladores de este sector? C) ¿Qué acciones realizan los pobladores que contribuyen a la contaminación en los parques lineales de la ciudad que están en las áreas cercanas a los ríos? D) ¿Qué medidas han tomado los vecinos de las áreas cercanas a los ríos para mitigar esta problemática?

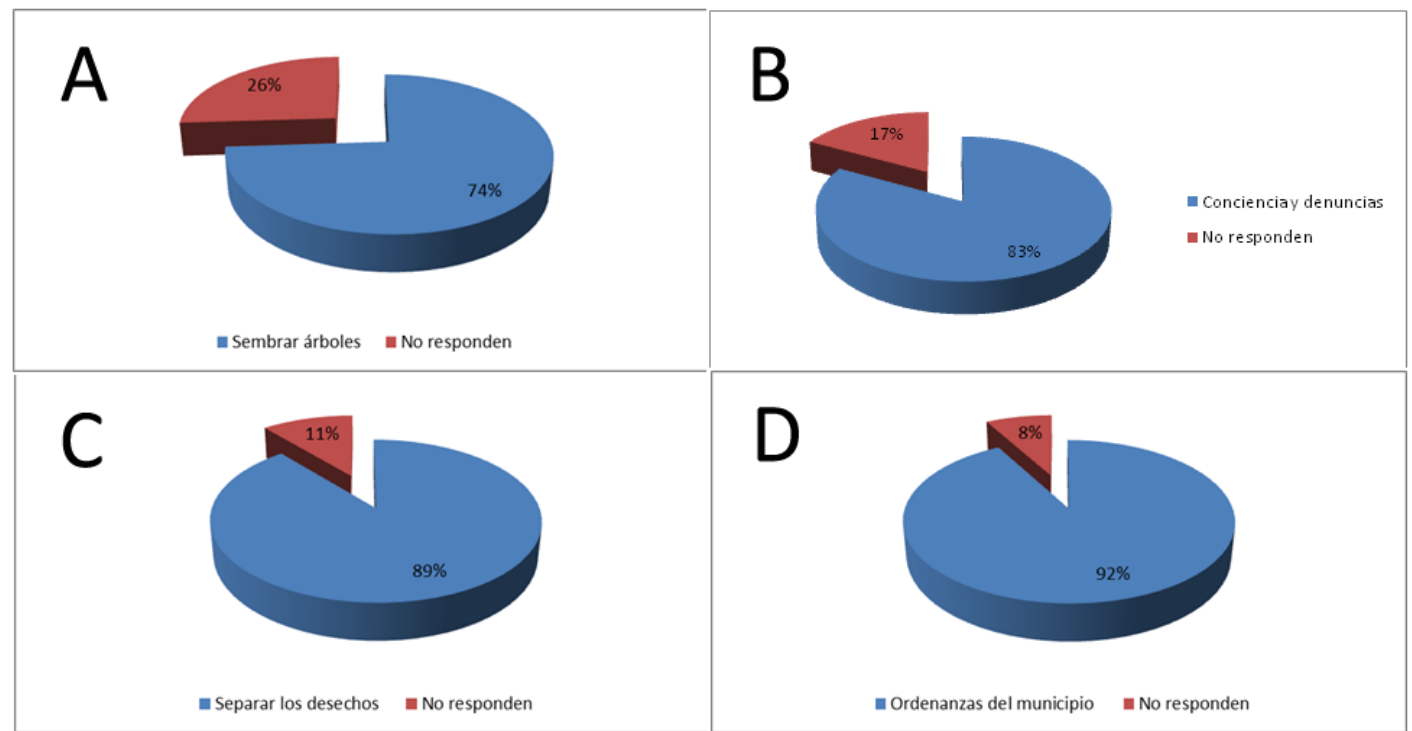

Figura 2. Proporciones de respuestas obtenidas a las siguientes preguntas: A) ¿Qué acciones de los vecinos para evitar la erosión de los suelos? B) ¿Qué acciones de los vecinos para evitar la tala de los árboles? C) ¿Qué acciones 
de los vecinos para el reciclaje de los desechos biodegradables y no degradables? D) ¿Qué acciones de los vecinos se pueden realizar para evitar la extracción de materiales pétreos?

A través del diagnóstico, se pudieron concretar las siguientes carencias y potencialidades:

Carencias:

- La existencia misma del problema ambiental.

- Las acciones de los pobladores que contribuyen a la contaminación de los ríos.

- No se han tomado medidas por parte de los vecinos de las áreas cercanas a los ríos para mitigar esta problemática.

- No han realizado los vecinos medidas para evitar la erosión de los suelos, la tala de árboles, para el reciclaje de los desechos, para evitar la extracción de materiales pétreos, entre otros.

- Desconocimiento de los vecinos de las áreas cercanas a los ríos sobre los daños que produce para la salud humana los efectos de la contaminación.

- No estar preparados para solucionar el problema.

- Inexistencia de proyectos de la comunidad dirigido a cambiar esta situación.

Potencialidades:

- Su reconocimiento sobre la existencia de contaminación de los ríos.

- Su dominio sobre cómo ocurre el proceso de contaminación de los ríos por parte de los pobladores del sector.

- Su conocimiento sobre algunas medidas de control a implementar por el Municipio para mitigar esta problemática.

- Su reconocimiento de no estar preparados para enfrentar esta problemática.

- Su disposición como líder a prepararse para cambiar esta situación

A través de los talleres y la posterior evaluación de los mismos, se permitió corroborar desde la metodología cualitativa aplicada un conjunto de logros esenciales alcanzados en la subjetividad de los sujetos comunitarios en transformación: se concienció por parte de los pobladores de las comunidades, sus fortalezas y debilidades para enfrentar el cambio; se construyó colectivamente el proyecto de autodesarrollo, dirigido a la formación en ellos de la cultura ambiental y la consciencia crítica necesaria para transformar el estado de cosas en la situación ambiental presentes en la zona de las microcuencas; se evidenció, a través del análisis del diario de campo, el significativo nivel de asimilación de los conocimientos y habilidades adquiridas en el tránsito de los mismos por el sistema de talleres, constatándose el dominio de conceptos medioambientales esenciales para la comprensión e interpretación de los mismos en su contexto de actuación, la incorporación de dichos contenidos a su vocabulario activo en los análisis de las problemáticas ambientales que enfrentan y la aplicación de los mismos en los análisis de disímiles situaciones analizadas.

\section{Discusión}


Del análisis realizado se observa que los habitantes de la microcuenca evaluada consideran la contaminación de los ríos como una problemática de origen antrópico ante la que no están preparados a afrontar, a pesar de conocer y proponer varias medidas de mitigación.

Estudios realizados en Argentina en las provincias Santiago del Estero, Salta y Jujuy y en varias provincias de Cuba por Rivero (2010) corroboran todos los resultados apuntados anteriormente, y nos llevan a reafirmar la eficacia de la aplicación del proyecto de intervención gestado desde la perspectiva del autodesarrollo, el cual estuvo dirigido a formar una cultura ambiental en los pobladores de las comunidades para potenciar la conciencia crítica de estos en la mitigación de los impactos negativos medioambientales vinculados al mal manejo de las microcuencas.

De acuerdo a los estudios realizados por Pérez (2016) en el trabajo comunitario realizado sobresalieron algunos rasgos como la disposición de la ciudadanía para apoyar las diferentes etapas de los proyectos que se elaboran y el compromiso asumido por las instituciones municipales sobre asesoría, recursos, sistematicidad de servicios etc., hubo participación y productividad y se retroalimentaron durante la aplicación comunitaria de cuidar su entorno natural para solucionar los problemas presentes y prevenir los futuros.

A lo largo del desarrollo de los talleres, se logró la adquisición de un grupo de habilidades esenciales para el análisis de las problemáticas ambientales, dentro de las que se encuentran: la habilidad de diagnosticar las situaciones puntuales asociadas a la problemáticas que subsisten en las microcuencas estudiadas, la habilidad asociada a analizar el sistema de contradicciones vivenciadas, las cuales se expresan como malestares de vida cotidiana, y la habilidad para encontrar de manera creativa soluciones endógenas a los problemas.

Al mismo tiempo, como resultado de las transformaciones operadas bajo el efecto del proyecto de transformación, se lograron vivenciar valores esenciales, importantes en los cambios de comportamientos y actitudes ante el medio ambiente como la responsabilidad, la solidaridad, el compromiso, la dedicación e implicación en las soluciones, entre otros.

Todos los resultados apuntados anteriormente nos llevan a reafirmar la eficacia de la aplicación del proyecto de intervención gestado desde la perspectiva del autodesarrollo, el cual estuvo dirigido a formar una cultura ambiental en los pobladores de las comunidades para potenciar la conciencia crítica de estos en la mitigación de los impactos negativos medioambientales vinculados al mal manejo de las microcuencas.

\section{Bibliografía}

Boff L. (2004). Saber cuidar: ética de lo humano, complejidad de la vida para la vida, 11. Ed. Petrópolis-RJ. Editora Vozes. 88 pp.

Colectivo de autores (2004a). Autodesarrollo Comunitario. Crítica a las mediaciones sociales recurrentes para la emancipación humana. Editorial Feijoo, Santa Clara. 
Colectivo de autores (2004b). La Educación Ambiental en la formación de docentes. Editorial Pueblo y Educación, La Habana.

Gonzaga A.G. (2014). Intervención comunitaria para desarrollar estrategias de mitigación de impactos contaminantes ambientales de las microcuencas de los ríos Malacatos y Zamora de la Hoya de Loja. Tesis de Doctorado en Ciencias Ambientales en la Universidad Nacional Piura, Perú.

Gonzaga, A. y Zúñiga, F (2015). Mapa de uso actual del suelo de la Microcuenca Jipiro.

Pérez, A (2016). Teoría y práctica del Desarrollo comunitario .Un estudio de caso en un fraccionamiento de Ciudad Juarez, Chiguagua. Universidad autónoma de ciudad Juárez.

Programa de las Naciones Unidas para el Medio Ambiente, Municipalidad de Loja y Naturaleza y Cultura Internacional. 2007

Revista Iberoamericana para la investigación y el desarrollo educativo.

Rivero R. (2010). Intervención Comunitaria, Familiar y de Género. Centro de Estudios Comunitarios Universidad Central "Martha Abreu” de Las Villas. UCLV: Editorial Feijóo.

ROQUE M. G. (2003). "Estrategia educativa para la formación de la cultura ambiental de los profesionales cubanos de nivel superior, orientada al desarrollo sostenible." Tesis presentada en opción al grado de Doctor en Ciencias de La Educación, La Habana.

SATO, Michèle. "Aguas e utopías tropicais. Contexto de projecto Mimoso, com resgate da cultura popular a través de lencas e mitos relacionados con a água". Disponible en http://www.ufmt.br/gpea/pub_artig.htm. Acceso en: 23 de septiembre de 2006.

Teixeira E.C., González H.M. y Urías G. (2008). Estrategia de superación profesional para la formación de la cultura ambiental de los docentes. Campo Verde. MTG. Brasil.

UNCSD, Conferencia de las Naciones Unidas sobre el Desarrollo Sostenible (2012). ¿Qué es Río+20? Disponible en: http://www.un.org/es/sustainablefuture/pdf/spanish_riomas20.pdf [consultado el 26/11/2016]

\section{Anexo 1. Modelo de encuesta realizada en la segunda fase de la metodología del desarrollo comunitario.}

1.- ¿Qué opina usted sobre la contaminación de los ríos?

2.- ¿Cómo ocurre el proceso de contaminación de los ríos por parte de los pobladores de este sector?

3.- ¿Qué acciones realizan los pobladores que contribuyen a la contaminación en los parques lineales de la ciudad que están en las áreas cercanas a los ríos?

4.- ¿Qué medidas han tomado los vecinos de las áreas cercanas a los ríos para mitigar esta problemática? 
5.- ¿Qué medidas de control debe implementar el Municipio para mitigar esta problemática?

6.-Explique que han hecho los vecinos para evitar la erosión de los suelos.

7.-Explique que han hecho los vecinos para evitar la tala de los árboles.

8.-Explique que han hecho los vecinos para el reciclaje de los desechos biodegradables y no degradables.

9.-Explique que han hecho los vecinos para evitar la extracción de materiales pétreos.

10.-¿Considera usted estar preparado para enfrentar esta problemática?

Si....... No......

Porqué.

11.- ¿Qué conocimientos poseen los vecinos de las áreas cercanas a los ríos sobre los daños que produce para la salud humana los efectos de la contaminación?

12.- ¿Qué conocimientos poseen los vecinos de las áreas cercanas a los ríos sobre los daños que produce para las aguas de los ríos los efectos de la contaminación?

13.- Está dispuesto usted como vecino a cambiar esta situación.

Si...... No....

Explique

14.-Existe algún proyecto en la comunidad dirigido a cambiar esta situación.

Si...... No....

Explique.

\section{Anexo 2. Talleres realizados en la cuarta fase de la metodología del desarrollo comunitario.}

Taller n $n^{o}$ 1: Presentación de la matriz para el diagnóstico participativo y para la búsqueda de soluciones.

\section{Objetivos:}

- Diagnosticar la información que tienen los habitantes de la Microcuenca Jipiro sobre los problemas ambientales que presenta su entorno y la responsabilidad que tienen ellos en la situación.

- Reflexionar con los grupos de pobladores de la comunidad sobre las posibles soluciones que ellos proponen para mitigar los impactos ambientales que se han originado en la Microcuenca donde habitan.

\section{Taller $n^{\circ}$ 2: Los problemas ambientales: concepciones generales}

\section{Objetivos:}

- Argumentar la importancia del conocimiento por parte de los asistentes de los principales problemas ambientales en el contexto global y local.

- Interpretar, a partir de un recorrido por el entorno de la microcuenca, los principales problemas ambientales globales que están afectando el entorno local.

Taller $n^{o}$ 3: Principales conceptos: medio ambiente, desarrollo sostenible, sociedad sostenible, manejo de residuos sólidos.

\section{Objetivos:}


- Argumentar la importancia del conocimiento, por parte de las personas de los principales conceptos relacionados con la problemática ambiental para lograr una cultura ambiental en los ciudadanos.

\section{Taller $n^{\circ} 4$}

\section{Tema: Contaminación por Residuos Sólidos y la importancia del reciclar}

Objetivo: Educar a los niños del barrio Jipiro Alto. Elaborar manualidades con botellas de plástico reutilizadas.

\section{Procedimiento:}

- Presentación de diapositivas a los niños con la temática: Contaminación por Residuos Sólidos.

- Presentación de videos relacionados con la temática tratada.

- Ronda de preguntas a los niños.

- Entrega de golosinas para los niños que respondieron correctamente.

- Elaboración de un portalápices con botellas plásticas reutilizadas.

- Entrega de premios a la mejor manualidad.

Taller $n^{\circ} 5$

\section{Tema: Minga en las inmediaciones del Barrio Jipiro Alto}

Objetivo: Motivar la participación de niños del barrio Jipiro Alto en la minga para recoger los residuos acumulados en las inmediaciones del barrio y en las riberas del Rio Jipiro

\section{Procedimiento:}

- Concentración de niños en la escuela del barrio.

- Presentación de diapositivas a los niños con la temática: Limpia tu comunidad.

- Entrega de implementos necesarios para la minga de limpieza del barrio, entre ellos: guantes y fundas de basura.

- Recolección de residuos sólidos inorgánicos en las inmediaciones del barrio y en las riberas del Rio Jipiro. 